\title{
Analysis on the MRI and BAEP Results of Neonatal Brain with Different Levels of Bilirubin
}

\section{Lu Zhongxing ( $\nabla$ szdxlzx@126.com )}

Changzhou Maternal And Child Health Care Hospital https://orcid.org/0000-0002-4383-1475

\section{Shouling Ding}

Paediatric Department of Taicang First People's Hospital

\section{Fen Wang}

Paediatic Department of Taicang First People's Hospital

Haitao Lv

Children's Hospital of Soochow University

\section{Research article}

Keywords: MRI abnormality change, acute bilirubin encephalopathy, bilirubin, hyperbilirubinemia, BAEP

Posted Date: December 23rd, 2020

DOl: https://doi.org/10.21203/rs.3.rs-17458/v3

License: (9) This work is licensed under a Creative Commons Attribution 4.0 International License. Read Full License 


\section{Abstract}

Background囚To explore whether there is abnormality of neonatal brains' MRI and BAEP with different bilirubin levels, and to provide an objective basis for early diagnosis on the bilirubin induced subclinical damage on brains.

Methods: To prospective study: analyze the clinical data of 103 neonatal patients, who had been hospitalized in Neonatology Department of Taicang First People's Hospital from March 2013 to September 2015, to conduct routine brain MRI examination , BAEP testing and to analyze BAEP and MRI image results of the neonatal patients, who were divided into three groups based on the levels of total serum bilirubin concentration (TSB), 16 cases in mild group (TSB:0.0 $229.0 \mu \mathrm{mol} / \mathrm{L}$ ), 49 cases in moderate group (TSB: 229.0 342.0 $\mu \mathrm{mol} / \mathrm{L}$ ) and 38 cases in severe group (TSB $\geq 342.0 \mu \mathrm{mol} / \mathrm{L})$;

Results: We found as follows: A. Comparison of the bilirubin value of the different group : 1 . The bilirubin value of the mild group is $171.99 \pm 33.50 \mu \mathrm{mol} / \mathrm{L}$, the moderate group is $293.98 \pm 32.09 \mu \mathrm{mol} / \mathrm{L}$, and the severe group is $375.59 \pm 34.25 \mu \mathrm{mol} / \mathrm{L}$. And the comparison of bilirubin values of the three groups of neonates (Pख0.01) indicates the difference is statistically significant (Pख0.01). 2. The bilirubin value of the pre-term group is $289.70 \pm 85.38 \mu \mathrm{mol} / \mathrm{Land}$ the full-term group is $310.36 \pm 72.32 \mu \mathrm{mol} / \mathrm{L}$, but the comparison of the bilirubin values between pre-term group and full-term group indicates that the difference is not statistically significant (Pख0.05).3. The bilirubin value of the normal brain MRI group(82) is $305.55 \pm 74.54 \mu \mathrm{mol} / \mathrm{L}$ and the abnormal brain MRI group is $303.56 \pm 83.04 \mu \mathrm{mol} / \mathrm{L}$; the comparison of bilirubin values between the two groups indicates that the difference is not statistically significant $(P \mathbb{Z}$ 0.05 ). B. The weight value of the $₫ 2500 \mathrm{~g}$ group is $2.04 \pm 0.21$ and the $\geq 2500 \mathrm{ggroup}$ is $3.39 \pm 0.46$; the weight comparison of the two groups indicates that the difference is statistically significant (Pख0.01). C. Comparison of the abnormal MRI of the different groups: 1.The brain MRI result's abnormal ratio of the mild group is $31.25 \%$, the moderate group is $16.33 \%$ and the severe group is $21.05 \%$, but the comparison of brain MRI results of the three neonates groups indicates that the difference is not statistically significant (P凶0.05). 2. The brain MRI result's abnormal ratio of the pre-term is $30.77 \%$ and the full-term group is $16.88 \%$, but the comparison of brain MRI results between prem-term group and full-term group indicates that the difference is not statistically significant (Pख0.05). 3.The brain MRI result's abnormal ratio of the $\mathbb{2} 2500 \mathrm{~g}$ group is $37.50 \%$ and the $\geq 2500 \mathrm{~g}$ group is $17.24 \%$; but the comparison of brain MRI results of two neonates groups indicates that the difference is not statistically significant(Pख0.05). D. Comparison of abnormal MRI signal values of globus pallidus on T1WI in different groups: 1 . The comparison of normal group signal values with that of mild group $(p<0.05)$, with that of moderate group and with that of severe group $(p<0.01)$ indicates that the difference is statistically significant; 2 . The comparison of signal values between mild and moderate groups $(p<0.05)$ and between mild group and severe group $(p<0.01)$ indicates that the difference is statistically significant; 3 . The comparison of signal values between moderate group and severe group indicates that the difference is statistically significant $(p<0.05)$. E. Comparison of BAEP testing results in groups: 1 . There were $27(26.21 \%)$ cases in abnormalities of the BAEP results of all 103cases bilirubin patients. 2. There were $15(18.29 \%)$ cases in abnormalities of the BAEP result of the 82 cases normal brain MRL ,2(40\%) cases in abnormalities of the 
BAEP result of the 5 cases abnormal MRI in mild bilirubin group, $4 \llbracket 50 \% \bigotimes$ cases in abnormalities of the

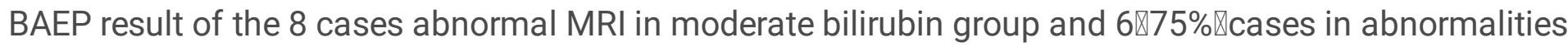
of the BAEP result of the 8 cases abnormal brain MRI in severe bilirubin group. 3. After one month review of the BAEP result, there was $0 \otimes 0.00 \% \triangle$ abnormal case in the normal MRI and the mild group; there were 1 $\triangle 20 \% \triangle$ abnormal case in the moderate group and $2 \varangle 25 \% \bigotimes$ cases in the severe group.

Conclusion: At low level of bilirubin, central nervous system damage may also occur and can be detected as abnormality by MRI and BAEP. Meanwhile, MRI and BAEP can also provide early abnormal information for the judgment of central nervous system damage of the children with NHB who have no acute bilirubin encephalopathy $(\mathrm{ABE})$ clinical features, and provide clues for early treatment and early intervention.

\section{Background}

Neonatal jaundice is quite common, affecting $60 \%-80 \%$ of newborns overall[ ${ }^{[1]}$. Mainly, with the high accumulation of unconjugated bilirubin, severe patients (with bilirubin concentration $>20 \mathrm{mg} / \mathrm{dL}$ ) may suffer from bilirubinencephalopathy or kernicterus, and bilirubun-induced neurological dysfunction $(B I N D)^{[2]}$, which will cause permanent damage to the basal ganglia, hippocampus, hypothalamus, cerebellar neurons, brainstem ${ }^{[3,4]}$, etc. Clinical manifestation includes lethargy, poor response, fever, apnea, convulsion, auditory complications and so on ${ }^{[2]}$. Magnetic resonance imaging (MRI) and hearing screening have proved to be highly sensitive in bilirubin encephalopathy. MRI can be used to detect highintensity signals of globus pallidus and subthalamic nucleus in early T1WI and high stripy symmetric signals of globus pallidus in later T2W ${ }^{[5,6]}$. And abnormal changes occur in the BAEP ${ }^{[7]}$. However, severe hyperbilirubinemia ( $>20 \mathrm{mg} / \mathrm{dL}$ ) that potentially leads to bilirubin encephalopathy or kernicterus and neurodevelopmental complications is said to be much rarer, affecting less than $2 \%$ of newborn infants ${ }^{[8]}$. Most of them have no clinical manifestation except jaundice. Whether a low level of hyperbilirubinemia causes brain damage to neonates is still under debate. It is also unclear whether different degrees of hyperbilirubinemia result in different patterns of brain injury. For answering these questions, we examined the brain MRI and hearing screening of neonates with different levels of bilirubin. All hospitalized neonates were tested daily for percutaneous bilirubin at 8 a.m. Serum bilirubin levels with abnormally elevated percutaneous bilirubin levels were tested.MRI and BAEP of all the children were examined on the third day after their bilirubin peak, and the children with abnormal results were reviewed within 1-3 months after birth in the Taicang First People's Hospital.

\section{Methods}

\subsection{Methods and Criteria}

\subsubsection{Methods}


MRI: A Siemens Skyra 3.0T superconductivity magnetic resonance scanner and a new Tim4G platform equipped with 20 units of high-density matrix head and neck joint coils were applied to this study. Before MRI scanning, the neonate patients were given routine sedation, kept in a supine position and warm. Other parameters include T1WI (TR=477 ms, TE=15 ms), T2WI (TR=4060 ms, TE=120 ms), a matrix of $256 \times 256$, a slice thickness of $5 \mathrm{~mm}$, and a gap of $0.4 \mathrm{~mm}$. Images were obtained and coronal scanning was done for all neonate patients.

\subsubsection{Criteria for Diagnosis}

All MRI results were jointly written and analyzed by two radiologists who did not know the clinical history of the patient.

MRI: high-intensity signals of globus pallidus and subthalamic nucleus detected in T1WI, high-intensity symmetric signals of globus pallidus detected in T2WI, or uneven signal of basal ganglion was considered abnormal. No above-mentioned manifestation was regarded as normality $[5,6]$.

\subsubsection{Brainstem Auditory Evoked Potential (BAEP)}

BAEP testing was performed by an otolaryngologist skilled in BAEP technology in a quiet environment.

\subsubsection{Criteria for Diagnosis}

BAEP: The results were considered to be abnormal when the incubation period of I wave, III wave and V wave was prolonged, the interphase period of I V wave was prolonged, the waveform was poorly differentiated or disappeared, or the single or bilateral I wave, III wave and $V$ wave all disappeared ${ }^{[7]}$.

\subsection{Statistical Method}

Data analysis was performed on SPSS 23.0 statistical software. Each measuring parameter was expressed by Mean $\pm S D$ and t-tests (multi-group comparisons), while each counting parameter was expressed by rate and $c^{2}$ tests (comparisons among groups). The statistically significant difference was identified by $\mathrm{P}<0.05$.

\section{Results}

\subsection{Clinical Data}

We prospective study: analyzed 103 neonates (51 boys and 52 girls), who were assumed to have no "clinical" manifestation of bilirubin encephalopathy, and were hospitalized in the Neonatology Department of Taicang First People's Hospital from March 2013 to September 2015. Their gestational ages ranged from $31+6$ weeks to $43+1$ weeks (on average, $38.14 \pm 2.21$ weeks) and birth weight ranged from $1.55 \mathrm{~kg}$ to $5.00 \mathrm{~kg}$ (on average, $3.18 \pm 0.64 \mathrm{~kg}$ ). They were composed of 26 pre-term neonates and 77 full-term neonates. These neonates were divided into three groups based on their levels of serum bilirubin 
concentration, 16 cases in the mild group (TSB: $0.0 \sim 229.0 \mu \mathrm{mol} / \mathrm{L}$ ), 49 cases in the moderate group (TSB: 229.0 342.0 $\mu \mathrm{mol} / \mathrm{L}$ ) and 38 cases in the severe group (TSB $\geq 342.0 \mu \mathrm{mol} / \mathrm{L})$. Possible diseases with similar manifestations to that of bilirubin encephalopathy, such as toxic cerebral hypoxia damage, hepatolenticular degeneration, hypoxic-ischemic encephalopathy, intracranial hemorrhage, hypoglycemia, etc. were excluded.

\subsubsection{Comparison of the three groups in terms of the bilirubin value}

Table 1

\begin{tabular}{|c|c|c|c|}
\hline Group & Bilirubin value & $t$ & $\mathrm{p}$ \\
\hline Mild group ${ }^{1}$ & $\underline{171.99} \pm 33.50$ & 20.54 & $\underline{0.00}$ \\
\hline Moderate group & $293.98 \pm 32.09$ & 64.13 & $\underline{0.00}$ \\
\hline Severe group & $\underline{375.59} \pm \underline{34.25}$ & $\underline{67.59}$ & $\underline{0.00}$ \\
\hline Pre-term $^{2}$ & $\underline{289.70} \pm 85.38$ & $\underline{-1.20}$ & 0.232 \\
\hline Full-term & $\underline{310.36 \pm 72.32}$ & & \\
\hline Normal MRI ${ }^{3}$ & $\underline{305.55 \pm 74.54}$ & $\underline{0.11}$ & $\underline{0.92}$ \\
\hline Abnormal MRI & $\underline{303.56 \pm 83.04}$ & & \\
\hline
\end{tabular}

1. The bilirubin values of the mild group, the moderate group, and the severe group are $171.99 \pm 33.50 \mu \mathrm{mol} / \mathrm{L}, 293.98 \pm 32.09 \mu \mathrm{mol} / \mathrm{L}$, and $375.59 \pm 34.25 \mu \mathrm{mol} / \mathrm{L}$, respectively, showing statistically significant differences $(\mathrm{P} 0.01)$. 2 . The bilirubin value of the pre-term group is $289.70 \pm 85.38 \mu \mathrm{mol} / \mathrm{Land}$ that of the full-term group is $310.36 \pm 72.32 \mu \mathrm{mol} / \mathrm{L}$, revealing no statistically significant differences ( $\mathrm{P} \square 0.05)$.3. The bilirubin value of the normal brain MRI group (82) is $305.55 \pm 74.54 \mu \mathrm{mol} / \mathrm{L}$ and that of the abnormal brain MRI group is $303.56 \pm 83.04 \mu \mathrm{mol} / \mathrm{L}$, implying no statistically significant differences ( $\mathrm{P} \square 0.05)$.

\subsubsection{Two groups divided by the weight of $2500 \mathrm{~g}$}

Table 2

\begin{tabular}{|l|l|l|l|l|l|l|l|l|l|}
\hline Group & & & $\mathbf{2} 200 \mathrm{~g}$ & & & & $\mathbf{2} \mathbf{2 5 0 0 \mathrm { g }}$ & & \\
\hline Weight & & $2.04 \pm 0.21$ & & & & $3.39 \pm 0.46$ & & \\
\hline $\mathrm{t}$ & & & & -18.85 & & & & \\
\hline $\mathrm{P}$ & & & 0.00 & & & & \\
\hline
\end{tabular}


The weight value of the $\square 2500 \mathrm{~g}$ group is $2.04 \pm 0.21$ and that of the $\geq 2500$ ggroup is $3.39 \pm 0.46$, indicating a statistically significant difference (P卬0.01).

\subsubsection{Comparison of the three groups in terms of abnormal MRI}

Table 3

\begin{tabular}{|l|l|l|l|l|}
\hline Group & Normal MRI & Abnormal MRI & $\mathrm{c}^{2}$ & $p$ \\
\hline Mild group & & & \\
\hline Moderate group & $41(68.75 \%)$ & $5(31.25 \%)$ & & \\
\hline Severe group & $30(78.95 \%)$ & $8(21.05 \%)$ & & \\
\hline & & & & \\
\hline Pre-term & & & & \\
\hline Full-term & $18(69.23 \%)$ & $8(30.77 \%)$ & 2.310 & 0.160 \\
\hline & $64(83.12 \%)$ & $13(16.88 \%)$ & & \\
\hline$<2500 g^{3}$ & & & & \\
$\geq 2500 g$ & $72(82.76 \%)$ & $15(17.24 \%)$ & & 0.09 \\
\hline
\end{tabular}

1.The brain MRI result's abnormal ratio of the mild group is $31.25 \%$, and those of the moderate group and the severe group are $16.33 \%$ and $21.05 \%$, respectively, implying no statistically significant differences (P॰0.05). 2. The brain MRI result's abnormal ratio of the pre-term is $30.77 \%$ and that of the full-term group is $16.88 \%$, implying no statistically significant differences (P־0.05). 3.The brain MRI result's abnormal ratio of the $\square 2500 \mathrm{~g}$ group is $37.50 \%$ and that of the of nd is RI result's aimplying no statistically significant differences (P匹0.05).

2.2.4 Comparison of mild, moderate and severe groups regarding abnormal MRI signal values of globus pallidus on T1WI and the comparison of abnormal with normal MRI signal values

Table 4

\begin{tabular}{|l|l|l|l|}
\hline Group & $\mathbf{n}$ & $\begin{array}{l}\text { Mean signal value of left globus } \\
\text { pallidus }\end{array}$ & $\begin{array}{l}\text { Mean signal value of right globus } \\
\text { pallidus }\end{array}$ \\
\hline Normal group ${ }^{1}$ & 82 & $892.03 \pm 132.54$ & $878.65 \pm 126.43$ \\
\hline Mild group $^{2}$ & 5 & $956.85 \pm 245.87$ & $942.06 \pm 232.60$ \\
\hline $\begin{array}{l}\text { Moderate group } \\
\text { 23 }\end{array}$ & 8 & $1056.23 \pm 254.21$ & $1032.32 \pm 222.32$ \\
\hline Severe group ${ }^{23}$ & 8 & $1246.03 \pm 278.46$ & $1239.03 \pm 288.53$ \\
\hline
\end{tabular}


1. When compared with the normal group in terms of the signal values, the mild group ( $\mathrm{p}<$ $0.05)$, the moderate group and the severe group ( $p<0.01)$ show statistically significant differences; 2 . The comparison of signal values between mild and moderate groups $(p<0.05)$ and between mild and severe groups $(p<0.01$ ) indicates statistically significant differences; 3 . The comparison of signal values between moderate and severe groups $(p<0.05)$ indicates a statistically significant difference.

\subsection{Comparison of BAEP testing results for neonates patients with abnormal MRI in three groups and comparison of hearing screening results between neonates patients with abnormal MRI and normal MRI.}

Table 5

\begin{tabular}{|c|c|c|c|c|}
\hline Group & $\begin{array}{l}\text { Normal } \\
\text { MRI } \\
\text { 082ロ }\end{array}$ & $\begin{array}{l}\text { Abnormal MRI in } \\
\text { mild group } 55 \square\end{array}$ & $\begin{array}{l}\text { Abnormal MRI in } \\
\text { moderate group } 80\end{array}$ & $\begin{array}{l}\text { Abnormal MRI in } \\
\text { severe group } \square 8 \square\end{array}$ \\
\hline Normal & 67 & 3 & 4 & 2 \\
\hline Abnormal & 15 & 2 & 4 & 6 \\
\hline$\%$ & 18.29 & 40.00 & 50.00 & 75.00 \\
\hline $\begin{array}{l}\text { Review abnormality } \\
\text { after one month }\end{array}$ & 0 & 0 & 1₫12.50凸 & $2 \square 25.00 \square$ \\
\hline
\end{tabular}

1. The BAEP results show 27 cases of abnormalities among all 103 cases of bilirubin patients. 2. The BAEP results show 15 cases of abnormalities among the 82 cases of normal brain MRL, 2 cases of abnormalities among the 5 cases of abnormal MRI in the mild bilirubin group, 4 cases of abnormalities among the 8 cases of abnormal MRI in the moderate bilirubin group and 6 cases of abnormalities among the 8 cases of abnormal brain MRI in the severe bilirubin group. 3. After one month review of the BAEP result, there was 0 abnormal case in the normal MRI and the mild group, 1 abnormal case in the moderate group and 2 cases in the severe group.

\subsection{MRI pictures}

(see Figure 1)

A normal $\mathrm{B}$ mild $\mathrm{C}$ moderate $\mathrm{D}$ severe $\mathrm{E}$ T2WI manifestation

A - A Newborn with a birth weight of $3.05 \mathrm{~kg}$, normal delivery at $36+3$ weeks and clear amniotic fluid, Apgar score of 1 '-9', 5 '-9'. The serum total bilirubin was $502.3 \mathrm{~mol} / \mathrm{L}$ on the 6 th day after birth. Routine MRI of the basal ganglia examined on the 9th day after birth showed an equal signal or slightly higher signal on $\mathrm{T} 1 \mathrm{WI}$, and the signal of globus pallidus was slightly higher than that of other nuclei in the basal ganglia on T1WI. 
B - A Newborn with a birth weight of $3.50 \mathrm{~kg}$, normal delivery at $40+4$ weeks and amniotic fluid囚pollution, Apgar score of 1'-9', 5 '-9'. The serum total bilirubin was $130.4 \mathrm{~mol} / \mathrm{L}$ on the 8 th day after birth. Routine MRI of the basal ganglia examined on the 9th day after birth showed a high signal on T1WI, and the signal of globus pallidus was symmetrically high on T1WI.

C - A Newborn with a birth weight of $3.25 \mathrm{~kg}$, normal delivery at $37+6$ weeks and clear amniotic fluid, Apgar score of 1 '-8', 5 '-9'. The serum total bilirubin was $278.3 \mathrm{~mol} / \mathrm{L}$ on the 5 th day after birth. Routine MRI of the basal ganglia examined on the 8th day after birth showed a high signal on T1WI, and the signal of globus pallidus was symmetrically high on T1WI.

D - A Newborn with a birth weight of $2.87 \mathrm{~kg}$, normal delivery at $38+5$ weeks and clear amniotic fluid, Apgar score of 1 ' -8 ', 5 '-8'. The serum total bilirubin was $478.6 \mathrm{~mol} / \mathrm{L}$ on the 7 th day after birth. Routine MRI of the basal ganglia on the 10th day after birth showed a high signal on $\mathrm{T} 1 \mathrm{WI}$, and the signal of globus pallidus was symmetrically high on T1WI.

E - A Newborn with a birth weight of $3.31 \mathrm{~kg}$, normal delivery at 40 weeks and clear amniotic fluid, Apgar score of 1 '-8', 5 '-9'. The serum total bilirubin was $327.3 \mathrm{~mol} / \mathrm{L}$ on the 7 th day after birth. Routine MRI of the basal ganglia at 10 postnatal days showed uneven signals in bilateral basal ganglia. Routine MRI of the basal ganglia revisited at 2 months showed high signals on $\mathrm{T} 2 \mathrm{WI}$, and the signal of globus pallidus was symmetrically high on T2WI.

\section{Discussion}

Neonatal jaundice is the most common clinical manifestation in the neonate period, which is mainly caused by the increase of serum bilirubin in neonates. In the prenatal period, due to the hypoxic environment, the number of red cells is increased and the hemoglobin content in the fetal is high. After birth, due to the hyperoxic environment, the survival time of red blood cells becomes short. The fetal hemoglobin is gradually converted to adult hemoglobin, producing a large amount of bilirubin, which exceeds the ability of the liver to treat bilirubin. When the bilirubin level is above $139 \mu \mathrm{mol} / \mathrm{L}$, skin or organ xanthochromia may appear, which is called neonate jaundice. It has been previously proposed that if the concentration of bilirubin in the blood is above $342 \mu \mathrm{mol} / \mathrm{L}$, serious damage to the nervous system of the neonates may occur, thus triggering the acute-stage manifestation of acute bilirubin encephalopathy $(A B E)$ or chronic kernicterus and BIND ${ }^{[9,10]}$. Brain damage caused by neonatal hyperbilirubinemia (NHB) is not always reversible, but may lead to cerebral palsy and hearing loss. The most common types of nerve injuries are the sequelae of severe neurological dyskinesia and hearing impairment ${ }^{[2,11-13]}$. Acute bilirubin encephalopathy or kernicterus and BIND mainly affects subcortical regions, such as globus pallidus, hypothalamus, substantia nigra, cerebellar dentate nucleus, hippocampus, brainstem, etc ${ }^{[14,15]}$. However, the underlying mechanisms are still unknown ${ }^{[16,17]}$. Most of the $A B E$ neonatal patients still have a chance to be recovered by timely treatment of reducing serum bilirubin levels, while only a few suffer from sequelae like kernicterus. 
The feature of MRI manifestation to bilirubin encephalopathy is the high signal of globus pallidus in the acute stage of T1-weighted imaging (T1WI). With the development of the disease, it changes from the high signal in the acute stage of T1WI to the high symmetric signal of bilateral globus pallidus and subthalamic nucleus in the chronic stage and FLAIR sequence signal ${ }^{[18-20]}$. In the neonatal period, the basal ganglia nerve cells have strong physiological and biochemical metabolism and oxygen consumption is increasing. Especially, the middle and later parts of the globus pallidus are the most sensitive ${ }^{[21]}$. This leads to the selective deposition of serum bilirubin in the globus pallidus, and the middle and later parts of the globus pallidus become more sensitive ${ }^{[21-23]}$. Besides, it will cause damage to neurons and glial cells, the apoptosis of neurons and the change of glial cells' mitochondria function ${ }^{[24,25]}$. Basal ganglia pallidus injury caused by hyperbilirubinemia can be efficiently detected by MRI. The high and symmetric signal of bilateral T1WI pallidal is an important imaging feature of neonatal $A B E$, while the change from a high signal of T1WI to a high signal of T2-weighted imaging (T2W1) is the imaging feature of nuclear jaundice on MRI, indicating neuronal cell necrosis and poor prognosis $^{[26]}$. Presently, the mechanism of MRI signal change remains unclear. This may be related to the reduction of the T1 value, which is caused by the deposition of bilirubin in glial cells and the destruction of bilirubin to the nerve cell membrane ${ }^{[27]}$.

Most of the MRI results mentioned above were obtained from brain MRI findings of the severe or profound NHB patients with bilirubin encephalopathy or non-bilirubin encephalopathy. So far, there is no report about whether the MRI results of mild and moderate NHB patients without bilirubin encephalopathy manifestation are abnormal. Therefore, this study retrospectively analyzes the bilirubin level and MRI results of 103 patients with jaundice to understand whether there is abnormal brain MRI at different bilirubin levels, as well as whether there is a statistical difference of abnormal brain MRI results in bilirubin levels, gestational age, and birth weight.

It is reported in the literature that some mild levels of bilirubin may cause temporary or permanent neurological sequelae under the condition that a certain level of bilirubin is considered safe by people ${ }^{[28]}$. Based on total serum bilirubin concentration (TSB), the 103 patients in our research were divided into three groups, including 16 cases in the mild group (TSB: $0.0-229.0 \mu \mathrm{mol} / \mathrm{L}$ ), 49 cases in the moderate group (TSB: $229.0-342.0 \mu \mathrm{mol} / \mathrm{L}$ ) and 38 cases in the severe group (TSB $\geq 342.0 \mu \mathrm{mol} / \mathrm{L}$ ). There were 21 cases with abnormal MRI results, including $5(31.25 \%)$ cases in the mild group, 8 (16.33\%) cases in the moderate group and $8(21.05 \%)$ cases in the severe group(Table 3$)$. The comparison of TSB among the three groups indicated that the difference was statistically significant (Pख0.01) (Table 1), whereas the comparison of abnormal brain MRI results among the three groups indicated that the difference was not statistically significant $(\mathrm{P} \otimes 0.05)$. This implies that bilirubin brain nerve damage may occur in patients without the manifestation of $\mathrm{ABE}$ even at a low level of bilirubin, and the rate of MRI abnormality does not grow with the rising bilirubin level. The study findings are not consistent with the reports of El Houchi, S.Z. et al. that the higher the total bilirubin level is, the higher the proportion of abnormal MRI is ${ }^{[29]}$. We think that the low number of cases in the mild group may also be an important factor. Under Taoka 140 et al.'s follow-up of the observed subjects for 2 years, it was found that the infants had been developing 
normally whether the high symmetric T1WI signal was demonstrated in globus pallidus (GP) and substantia niga (STN) regions or not. In accordance with reports in the literature, it may be related to the development of gray matter mass in GP and STN after the birth of neonates ${ }^{[30-33]}$. Besides, substantial research has reported that this manifestation is one of the brain MRI findings of nuclear jaundice ${ }^{[34-36]}$. Along with the extensive clinical application of MRI, the manifestation is becoming more and more common in neonates including normal neonates. Thus, some scholars recently have raised different opinions ${ }^{[29-31]}$. For example, Harris et al. ${ }^{[37]}$ reported that a high symmetric signal in the globus pallidus region of four 5-21 day neonates with acute kernicterus disappeared during the follow-up. This manifestation was thought transient, showing no correlation with the prognosis of the patients. This manifestation also appeared in the cases of neonatal hypoxic-ischemic encephalopathy, hypoglycemia and other cases ${ }^{[38,39]}$.

In this study, the neonate patients, with a high signal of globus pallidus in T1WI or T2W1 caused by the diseases of neonatal hypoxic-ischemic encephalopathy, hypoglycemia, hepatolenticular degeneration, or other diseases, were excluded. Of our 21 patients with MRI, 12 were followed up for one month, and 9 cases were followed up for 3 months. Moreover, 6 cases of patients had normal brain MRI results in the reexamination of one month later. And 3 cases of patients were not reexamined of the MRI, one with neurological abnormalities 2 months later, who was delivered with a gestational age of 40 weeks, a birth weight of $3.31 \mathrm{~kg}$, clear amniotic fluid, an Apgar score of 8 at 1 min and 9 in 5 min, a maximum bilirubin Value of $327.3 \mu \mathrm{mol} / \mathrm{L}$, and an uneven signal of the brain MRI in bilateral basal ganglia region. However, one case of this study showed no brain MRI abnormalities with a maximum bilirubin value of 502.3 $\mu \mathrm{mol} / \mathrm{L}$, a birth weight of $3.05 \mathrm{~kg}$, a gestational age of $36+3$ weeks, clear amniotic fluid and an Apgar score of 9 at $1 \mathrm{~min}$ and 9 in $5 \mathrm{~min}$. In addition, another case in the study may have ABE with a gestational age of $40+4$ weeks, a birth weight of $3.5 \mathrm{~kg}$, meconium-stained amniotic fluid during birth, an Apgar score of 9 at $1 \mathrm{~min}$ and 9 in $5 \mathrm{~min}$, the maximum bilirubin value of $130.4 \mu \mathrm{mol} / \mathrm{L}$ and a high symmetric T1WI signal of bilateral basal ganglia indicated by his brain MRI. The high symmetric signal of bilateral globus pallidus is not unique to the neonatal $A B E$, but can also be seen in some neonatal patients with hypoxicischemic encephalopathy ${ }^{[40,41]}$, or even in normal neonates. However, the MRI manifestations of hypoxicischemic encephalopathy (HIE) involved a more extensive scope, which was characterized by internal capsule, the putamen and the thalami, and accompanied by cortical and subcortical, deep leukoplakia plaque abnormal signals, diffuse brain edema, intracranial hemorrhage, and so on. These accompanied manifestations were rarely reported in bilirubin encephalopathy ${ }^{[42]}$.

Blood-brain barrier injury might be caused by anoxia or other factors in antepartum and intrapartum, increasing the permeability of the blood-brain barrier and causing more free bilirubin to enter into brain tissue through the injured blood-brain barrier and to deposit in the basal nerve nucleus, cerebral ganglia, subthalamic nucleus, parietal nucleus, ventricular nucleus, caudate nucleus, cerebellum, oblongata, cerebral cortex, spinal cord and brainstem, etc ${ }^{[43]}$. Accordingly, the utilization of oxygen in brain tissue was inhibited, 176 leading to brain damage. It is also believed that bilirubin deposition causes the influx of neuron cells Ca2+ ${ }^{[44]}$, stimulates the increase of proteolytic enzyme activity ${ }^{[45]}$, oxidative damage ${ }^{[46]}$, 
immune stimulation, immunotoxicity ${ }^{[47]}$, increased cytoglutamate and excitotoxicity ${ }^{[48]}$, inflammatory damage ${ }^{[49]}$ and other pathways, leading to neuronal necrosis and apoptosis ${ }^{[41]}$. It has been reported in many studies that the occurrence of neonatal BE is related to such factors as gestational age ${ }^{[50]}$, birth weight ${ }^{[51]}$, bilirubin binding state and bilirubin level ${ }^{[52]}$, etc. In this study, 103 patients were divided into the pre-term group ( 26 cases) and the full-term group (77 cases), for the comparison of their bilirubin values (289.70 $\pm 85.38 \mathrm{vs} 310.36 \pm 72.32, P=0.232)$ and $M R I$ abnormal result $(P=0.16)$, showing no statistically significant difference (Pख0.05). It indicated that there may be no difference in the toxicity of bilirubin to the central nervous system between the pre-term group and the full-term group without BE clinical manifestation. Moreover, premature infants with younger gestational age appeared not to be more susceptible to bilirubin toxicity. The explanations of this result may be as follows: (1) Premature delivery patients, who are usually admitted to the hospital after birth, are treated for jaundice in time during hospitalization; while full-term infants are admitted to the hospital only when their bilirubin value reaches a higher level. On the one hand, a high level of bilirubin can easily cause nerve damage by passing through the blood-brain barrier; on the other hand, the longer the high level of bilirubin remains in the body, the more neurotoxicity the bilirubin is ${ }^{[53]}$. (2) The statistical analysis in this study may be biased, on account of the fewer cases in the pre-term delivery group, especially the lack of pre-term patients less than 31 weeks of gestational age due to obstetric factors. Meanwhile, we also observed whether there was a statistical difference in birth weight and brain MRI abnormality among jaundiced patients. Based on birth weight, 103 patients were divided into two groups, one group with a birth weight of $<2500 \mathrm{~g}$ ( 16 cases, including 6 abnormal cases) and the other group with a birth weight of $\geq 2500 \mathrm{~g}$ (87 cases, including 15 abnormal cases) (Table 2). The comparison of brain MRI abnormality between the two groups was $P=0.09, P>0.05$, indicating that the difference was not statistically significant.

In addition, the comparison of the bilirubin value between the abnormal MRI group and the normal MRI group was $303.56 \pm 83.04$ vs $305.55 \pm 74.54(P=0.92, P>0.05)$ (Table 1$)$. The difference was not statistically significant, which indicated that there was no obvious difference in the bilirubin level between the abnormal MRI group and the normal MRI group of jaundiced patients without ABE.

Moreover, we measured the T1WI signal values of the patients with abnormal brain MRI and the signal values of the patients with normal brain MRI in the three groups, and performed statistical comparison. The results showed that the T1WI signal values of the patients with abnormal MRI were all higher than those of the patients with normal MRI $(P<0.05)$, and the T1WI signal values of the patients with MRI abnormalities in the three groups also had statistically difference $(P<0.05)$ (Table 4). With the increase of serum bilirubin level, the T1WI signal value of the patients with MRI abnormalities also increased, which was consistent with the report of Yan Ruifang et al ${ }^{[54]}$ that there was a linear correlation between the mean signal value of globus pallidus and serum total bilirubin levels in the lesion group. This indicated that with the increase in the bilirubin level, the more bilirubin deposited on neuron such as globus pallidus, the more serious damage would be made to nerve tissue such as neuron. 
NHB can cause not only acute bilirubin encephalopathy and kernicterus but also bilirubin induced neurological dysfunction (BIND) ${ }^{[55,56]}$, which includes mild neurological abnormalities, cognitive disorder, auditory neuropathy spectrum disorder (ANSD) ${ }^{[57]}$ and so on. Auditory complications, a disabling neurological finding in kernicterus, are typically characterized by varying degrees of auditory neuropathy/dys-synchrony (AN/AD) ranging from central auditory processing difficulties with normal hearing to severe AN/AD with absent auditory brainstem responses, and possibly accompanying severe hearing loss and deafness. In fact, the brainstem cochlear nuclei are said to be one of the first structures affected by elevated total bilirubin, followed by the auditory nerve ${ }^{[58,59]}$. Thus, we performed hearing tests for the 103 patients with brainstem auditory evoked potential (BAEP) ${ }^{[60]}$ devices. From Table 5, it was found that 15 cases of 82 patients with normal MRI results were abnormal, accounting for $18.29 \%$. In the mild group, 2 cases of 5 patients with abnormal MRI were abnormal, accounting for $40.00 \%$. In the moderate group, 4 cases of 8 patients with abnormal MRI were abnormal, accounting for $50.00 \%$. In the severe group, 6 cases of 8 patients with abnormal MRI were abnormal, accounting for $75.00 \%$. The change of BAEP is closely related to bilirubin concentration and duration, but BAEP may be abnormal in children with a moderate bilirubin level. In other words, BAEP may be abnormal in children with related safe bilirubin abnormalities that are improved after clinical treatment, even if there is no clinical manifestation ${ }^{[61,62]}$. BAEP was improved with the decrease of serum bilirubin or after the process intervention such as phototherapy and blood exchange transfusion ${ }^{[63-65]}$. Vitoria Akinpelu, et al[ ${ }^{[61]}$. reported in 2013 that the abnormal BAEP rate fluctuated between $9 \%$ and $83.3 \%$ before the clinical intervention, and about over half of the BAEP abnormalities were improved after clinical treatment. After admission, all 103 patients were treated with medication and/or phototherapy, and their jaundice decreased significantly. After a 1-3 month followed-up, the reexamination was carried out, showing that the patients with hearing abnormalities in the MRI normal group and the mild group all returned to the normal level, while 1 case in the moderate group and 2 cases in the severe group remained abnormal. Sharma ${ }^{[66]}$, et al. and Agrawal ${ }^{[67]}$, et al. showed that BAEP improved in $77.2 \%$ and $76.5 \%$ of the children surveyed. This result indicated that on the one hand, the BAEP abnormal rate of the patients with abnormal MRI was higher than that of the patients with normal MRI, and the BAEP abnormal rate of the patients with abnormal MRI increased with the rising bilirubin level. It also showed that with the increase of bilirubin, the hearing damage became more obvious ${ }^{[68]}$. On the other hand, it proved the application value of MRI in neonatal jaundice patients, which could timely detect the abnormalities of the central nervous system of the patients with jaundice and provide imaging evidence for early diagnosis and early intervention.

Based on this study, we found abnormalities in the neonatal brain's MRI and BAEP at different bilirubin concentration levels. It indicates that in some conditions, even a low level of bilirubin concentration might lead to neurological damage in the neonatal period. Therefore, strengthening the monitoring of neonatal jaundice and using the MRI and BAEP to test the bilirubin induced neurological damage for neonates are recommended.

\section{Limitations of the Present Study}

Page 12/19 
Although the findings of this study indicate that in babies with jaundice, the brain MRI may show abnormal changes at mild and moderate levels of bilirubin, and there is no statistically significant difference between brain MRI abnormalities in the severe group, our results have been affected by some issues. First, we failed to measure the free bilirubin levels, internal environmental conditions such as serum albumin levels, $\mathrm{pH}$ values, etc. of the jaundice newborns participating in the study at that time. Therefore, it cannot be said with certainty that the baby's brain was exposed to bilirubin crossing the blood-brain barrier Prime type. Secondly, the sample size in this study is relatively small, especially in the mild group and the group with a birth weight of less than $2500 \mathrm{~g}$. Finally, the craniocerebral pathology test of the newborn animal Jaundice model and long-term follow-up was not performed to validate our findings. This issue should be addressed in future research.

\section{Conclusion}

In conclusion, in the presence of certain factors, such as premature, hypoproteinemia, potential intrauterine hypoxia, etc., central nervous system damage may also occur at a low level of bilirubin and result in an abnormality on MRI and BAEP. Meanwhile, MRI and BAEP can also be used to provide early abnormal information for the judgment of central nervous system damage to NHB neonatal patients without clinical manifestations of $\mathrm{ABE}$, and offer clues for early treatment and early intervention to prevent the occurrence of severe brain tissue damage.

\section{Abbreviations}

ABE: acute bilirubin encephalopathy

TSB: total serum bilirubin

MRI: magnetic resonance imaging

NHB: neonatal hyperbilirubinemia

T1Wl: T1-weighted imaging

T2Wl: T2-weight imaging

BAEP: brainstem auditory evoked potential

ANSD : auditory neuropathy spectrum disorder

BIND: bilirubin induced neurological dysfunction

AN/AD : auditory neuropathy/dys-synchrony

\section{Declarations}


Availability of data and material

The datasets generated and/or analysed during the current study are not publicly available due to regulation by the hospital.

Consent to publish: Our authors agree to publish this article.

Competing interests: The authors claim no conflict of interest.

Funding: There is no funding source.

\section{Authors' Contributions:}

Lu: Responsible for project writing/execution and paper writing.

D and W: Participate in project execution and data collection.

Lv: Give guidance on this subject and revise the paper.

All authors have read and approved the manuscript.

Acknowledgements: We would like to express our heartfelt thanks to all our colleagues for their assistance in this subject.

\section{References}

1. Olusanya, B.O.; Ogunlesi, T. A.; Slusher, T. M. Why is kernicterus still a major cause of death and disability in low-income and middle-income countries[J]? Arch. Dis. Child. 2014,99,1117-1121.

2. Shaprio, S.M. Chronic bilirubin encephalopathy: Diagnosis and outcome[J]. Semin. Fetal Neonatal Med. 2010,15, 157-163.

3. Uziel, A.; Marot, M.; Pujol, R. The Gunn rat: An experimental model for central deafness[J]. Acta Otolaryngol.1983, 95, 651-656.

4. Olds, C.; Oghalai, J.S. Bilirubin-Induced Audiologic Injury in Preterm Infants[J]. Clin. Perinatol. 2016, 43,313-323.

5. Wiskownski, J.L.; Panigraphy, A.; Painter, M.J.; et al.Magnetic resonance imaging of bilirubin encephalopathy: Current limitations and future promise[J]. Semin. Perinatol. 2014, 38, 422-428.

6. Okumura A. Hayakawa F. Maruyama K, et al. Single photo emission computed tomography and serial MRI in preterm infants with kernicterus[J]. Brain Dev, 2006,28(6): 348-352.

7. N Y Boo M. Oakes M S. Lye H. Said, Risk factors associated with hearing loss in term neonates with hyperbilirubinaemia[J], J. Trop. Pediatr, 1994, 40:194-197.

8. Muchowski, K.E. Evaluation and treatment of neonatal hyperbilirubinemia[J]. Am. Fam. Physician $2014,89,873-878$. 
9. Olusanya, B.O.; Ogunlesi, T.A.; Slusher, T.M. Why is kernicterus still a major cause of death and disability in low-income and middle-income countries[J]? Arch. Dis. Child. 2014, 99, 1117-1121.

10. Olusanya, B.O.; Kaplan, M.; Hansen, W.R. Neonatal hyperbilirubinemia: A global perspective[J]. Lancet Child Adolesc. Health 2018, 2, 610-620.

11. Shapiro SM. Kernicterus. In: Stevenson DK, Maisels MJ,Watchko JF, editors. Care of the Jaundiced Neonate [J].New York: McGraw-Hill; 2012. p. 229-242.

12. Shapiro SM, Popelka GR. Auditory Impairment in Infants at Risk for Bilirubin-induced Neurologic Dysfunction[J]. Semin Perinatol. 2011; 35: 162-170.

13. Campistol J, Galvez H, Cazorla AG, et al. Neurological Dysfunction Induced by Bilirrubin[J]. Neurologia, 2012; 27: 202-211.

14. López-Corella, E.; Ibarra-González, l.; Fernández-Lainez, C.; et al. Kernicterus in a boy with ornithine transcarbamylase defificiency: A case report[J]. Neuropathology 2017, 37, 586-590.

15. Adle-Biassette, H.; Harding, B.; Golden, J.A. Developmental Neuropathology; John Wiley and Sons Ltd.: Oxford, UK, 2018.

16. Watchko JF, Painter MJ, Panigrahy A. Are the Neuromotor Disabilities of Bilirubin-induced Neurologic Dysfunction Disorders Related to the Cerebellum and Its Connections? [J] Semin Fetal Neonatal Med 2015, 20: 47-51.

17. Wisnowski JL, Panigrahy A, Painter MJ, et al. Magnetic Resonance Imaging of Bilirubin Encephalopathy: Current Limitations and Future Promise[J]. Semin Perinatol 2014;38:422-428.1

18. Parashari UC, Singh R, Yadav R, et al. Changes in the Globus Pallidus in Chronic Kernicterus [J]. J Pediatr Neurosci 2009;4: 117-119

19. Magalha es MOGM, Brası 'lia DF. Kernicterus, Chronic Bilirubin Encephalophy: Imaging Features and Clinical Correlation[J]. ECR2016/ C-2261.

20. Ribeiro BN, Lima GA, Ventura N, et al. Chronic Kernicterus: Magnetic Resonance Imaging Findings[J]. Radio Bras 2016;49:407e8.

21. Ribeiro BN, Lima GA, Ventura N, Gasparetto EL, Marchiori E. Chronic Kernicterus: Magnetic Resonance Imaging Findings[J]. Radio Bras 2016;49:407e8.

22. Cece $H$, Abuhandan $M$, Cakmak A,et al. Diffusion-weighted Imaging of Patients with Neonatal Bilirubin Encephalopathy[J]. Jpn J Radiol 2013;31:179-185.

23. Gkoltsiou K, Tzoufi M, Counsell S, et al. Serial Brain MRI and Ultrasound Findings: Relation to Gestational Age, Bilirubin Level, Neonatal Neurologic Status and Neurodevelopmental Outcome in Infants at Risk of Kernicterus[J].Early Hum Dev 2008;84:829-838

24. Brito,M.A.,Silva,R.F.,andBrites, D.Bilirubin induces loss of membrane lipids and exposure of phosphatidylserine in humanery- throcytes[J]. CellBiol.Toxicol. 2002,18, 181-192.

25. Falcão,A.S.,Silva,R.F.,Pancadas, S., et al. Apoptosisand impairment of neuritenet work by short exposure of immature ratcor- tical neuron stoun conjugated bilirubin increase with cell differentiation 
and are additionally enhanced by an inflammatory stimulus[J]. J. Neurosci. Res.2007, 85, 12291239.

26. Okumura Akihisa, Kidokoro Hiroyuki, Shoji Hiromichi, et al. Kernicterus in preterm infants[J]. Pediatrics, 2009, 123 (6): e1052-1058.

27. Amit Y, Poznansky MJ, Schiff D: Neonatal jaundice and bilirubin encephalopathy: a clinical and experimental reappraisa[J]I. Isr J Med Sci 1992, 28:103-108.

28. Govaert $P$, Lequin $M$, Swarte R, et al. Changes in Globus Pallidus with (pre)term Kemicterns [J].Pediatrics, 2003, 112(6 Pt 1)|1256 -1263\

29. El Houchi, S.Z.; Iskander, I.; Gamaleldin, R.; et al. Prediction of 3- to 5-Month Outcomes from Signs of Acute Bilirubin Toxicity in Newborn Infants[J]. J. Pediatr. 2017, 183, 51-55.

30. Taoka T, Aida N, Ochi T, et al. Transient Hyperinteusity in the Subthalamic Nucleus and Glohus Pallidus of Newborns on T1-weighted images [J]. AJNR Am J Neuroradiol, 2011, 32(6):1130-1137区

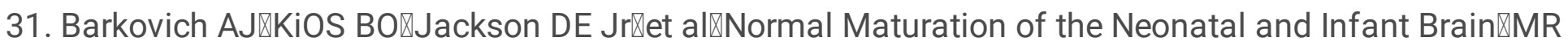
Imaging at 1.5T [J]. Radiology, 1988, 166(1 Pt 1):173-180.

32. Counsell SJ,Maalouf EF, Fletcher AM, et al. MR Imaging Assessment of Myelination in the Very Preterm Brain [J]. AJNR Am J Neuroradiol, 2002,23(5):872-881.

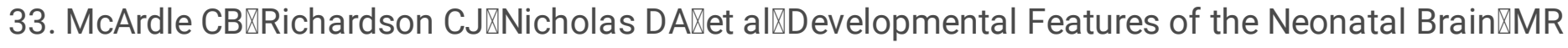
Imaging. Part IखGray-white Matter Differentiation and Myelination [J]. Radiology, 1987, 162(1 Pt 1):223-229ه

34. Penn AA, Enzmann DR, Hahn IS, et al. Kemictems in a Full-term Infant [J]. Pediatrics, 1994, 93(6 Pt 1):1003-1006ه

35. Govaert P, Lequin M, Swarte R, et al. Changes in Globus Pallidus with (pre)term Kemicterns [J].Pediatrics, 2003, 112(6 Pt 1)|1256 -1263】

36. Coskun A, Yikilmaz A, Kumand $\infty S$, et al. Hyperintense Globus Pallidus on T1-weighted MR Imaging in Acute Kernicterus: Is It Common or Rare? [J]. Eur Radial, 2005, 15(6):1263-1276区

37. Harris MC『Bembaum JC『Polin JR囚et al. Developmental Followup of Breastfed term and Near-term Infants with Marked Hyperbilirubinemia [J]. Pediatrics, 2001, 107(5):1075-1080

38. De Vries LS, Groenendaal F. Patterns of Neonatal Hypoxic Ischaemic Brain Injury [J]. Neumradiology, 2010, 52(6):555-566》

39. Bekiesinska. Figatowska M, Mierzewska H, et al. Basal Ganglia Lesions in Children and Adults [J]. Eur J Radiol, 2013, 82(5):837-849

40. Cece H, Abuhandan M, Cakmak A, et al. Diffusion-Weighted Imaging of Patients with Neonatal Bilinlbin Encephalopathy [J].Jpn J Radiol, 2013, 31(3):179-185

41. Barkovich J『MR of the neonatal neonatal bmin: Assessment of Deep stlllctures [J]区 AJNR囚1998】 19(8): 1397-1403.

42. K. Kaga, M. Suzuki, S. Koyama, Neonatal asphyxia and hyperbilirubinemia[J].JOHNS 16 (2000) 1695 -1699 . 
43. .Nakamura $H$, Takada $S$, Shimabuku $R$, et al. Auditory nerve and brainstem responses in newborn infants with hyperbilirubinemia[J]. Pediatrics, 1985, 75:703-708.

44. Zhang, B., Yang, X., and Gao, X. . Taurine protects against bilirubin induced neurotoxicity in vitro[J]. Brain Res. 2010,1320, 159-167.

45. Vaz, A. R., Silva, S. L., Barateiro, A., et al. Proinflflammatory cytokines intensify the activation of NO/NOS, JNK1/2 and caspase cascades in immature neurons exposed to elevated levels of unconjugated bilirubin[J]. Exp. Neurol.2011, 229, 381-390.

46. Hansen, T. W., Allen, J. W., and Tom marello, S. (1999). Oxidation of bilirubin in the brain-further characterization of a potentially protective mechanism[J]. Mol. Genet. Metab. 68, 404-40

47. Falcão, A. S., Fernandes, A., Brito, M. A., et al. Bilirubin-induced immunostimulant effects and toxicity vary with neural cell type and maturation state[J]. Acta Neuropathol. 2006,112, 95-105.

48. Silva, S. L., Vaz, A. R., Diógenes, M. J., et al. Impairment of neurite sprouting by unconjugated bilirubin is mediated by NO and glutamate,modulated by microglia, and prevented by glycoursodeoxycholic acid and interleukin-10[J]. Neuropharmacology, 2012, 62, 2397-2407.

49. Fernandes, A., Falcão, A. S., Silva, R. F.,et al. Inflflammatory signalling pathways involved in astroglial activation by unconjugated bilirubin[J]. J. Neurochem.2006, 96, 1667-1679.

50. Muchowski KE. Evaluation and Treatment of Neonatal Hyperbilirubinemia[J]. Am Fam Physician. $2014 ; 89(11): 873-878$.

51. Vidit Bhargava, Daniel Tawfik, Bruce Niebuhr, et al. Transcutaneous Bilirubin Estimation in Extremely Low Birth Weight Infants Receiving Phototherapy: A Prospective Observational Study[J]. BMC Pediatr ,2018; 18 (1) :227.doi: 10.1186/s12887-018-1207-7.

52. El Houchi, S.Z.; Iskander, I.; Gamaleldin, R.;et al. Prediction of 3- to 5-Month Outcomes from Signs of Acute Bilirubin Toxicity in Newborn Infants[J]. J. Pediatr. 2017, 183, 51-55.

53. Gkoltsiou,K.,Tzoufi,M.,Counsell, S.,et al. Serial brain MRI and ultrasound findings:relation to gestational age, bilirubinlevel, neonatal neurologic status and neurodevelopmental outcome in infants at risk of kernicterus[J]. EarlyHum .Dev. 2008, 84, 829-838.;

54. Zhou Liu, Bing Ji,Yuzhong Zhang,et al.Machine Learning Assisted MRI Characterization for Diagnosis of Neonatal Acute Bilirubin Encephalopathy[J].Front Neurol. 2019; 10: 1018. doi: 10.3389/fneur.2019.01018

55. Shapiro SM. Definition of the clinical spectrum of kernicterus and bilirubin induced neurological dysfunction (BIND)[J]. J Perinatol,2005, 25 (1): 54-59.

56. Smitherman H, Stark AR, Bhutan VK.Early recognition of neonatal hyperbilirubinemia and its emergent management[J]. Semin Fetal Neonatal Med, 2006, 11(3): 214-224.

57. Rapin I, Gravel J. "Auditory neuropathy” 『physiologic and pathologic evidence calls for more diagnostic specificity[J]. Int J Pediatr Otorhinolaryngol, 2003,67(7): 230-246.

58. Uziel, A.; Marot, M.; Pujol, R. The Gunn rat: An experimental model for central deafness[J]. Acta Otolaryngol.1983, 95, 651-656. 
59. Olds, C. Oghalai, J.S. Bilirubin-Induced Audiologic Injury in Preterm Infants[J]. Clin. Perinatol. 2016, 43,313-323.

60. Kimitaka Kaga , Eiji Kitazumi , Kazuo Kodama. Sensorineural hearing loss in patients with cerebral palsy after asphyxia and hyperbilirubinemia [J]. International Journal of Pediatric Otorhinolaryngology (2005) 69, 1211-1217

61. Olubunmi Victoria Akinpelu, Sofia Waissbluth, Sam J. Daniel. Auditory risk of hyperbilirubinemia in term newborns: A systematic review international[J]I Journal of Pediatric Otorlaryngology, 2013,77:898-905.

62. K. Sheykholeslami K. Kaga. Otoacoustic emission and auditory response after neonatal hyperbilirubinemia[J], Int. J. Pediatr. Otorhinolaryngol, 2000,52:65-73.

63. Perlman $\mathrm{M}$, Fainmesser $\mathrm{P}$, Sohmer $\mathrm{H}$, et al. Auditory nerve-brainstem evoked response in hyperbilirubinemic neonates[J]. Pediatrics, 1983, 72:658-664.

64. Nwaesei CG, Van Aerde J, Boyden M, et al. Changes in auditory brainstem responses in hyperbilirubinemic infants before and after exchange transfusion[J]. Pediatrics, 1984, 74(5): 800803.

65. Wennberg RP, Ahlfors CE, Bickers R, et al. Abnormal auditory brainstem response in a newborn infant with hyperbilirubinemia: improvement with exchange transfusion[J]. J Pediatr, 1982, 100 (4): 624626.

66. V. K. Agrawal, R. Shukla, P. K. Misra, R. K. Kapoor, G. K. Malik, Brainstem auditory evoked response in newborns with hyperbilirubinemia[J], Indian Pediatr, 1998, 35: 513-518.

67. P. Sharma NP. Chhangani KR. Meena R, et al. Gupta, Brainstem evoked response audiometry (BAER) inneonates with hyperbilirubinemia[J], Indian J. Pediatr, 2006, 73: 413-416.

68. Funato M, Tamai H, Shimada S, et al. Vigitiphobia, unbound bilirubin, and auditory brainstem responses[J]. Pediatrics, 1994,93(1): 50-53.

\section{Figures}



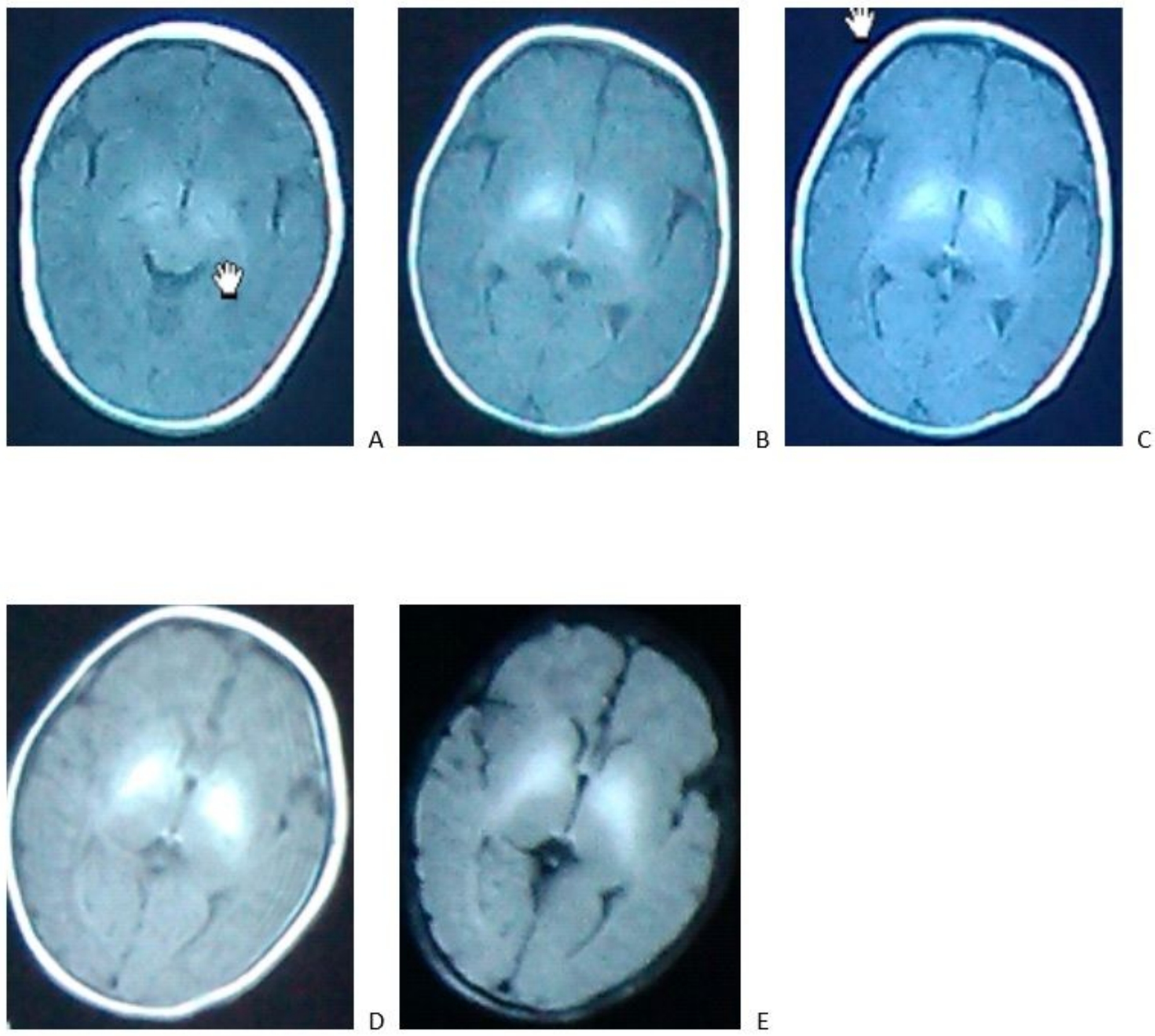

\section{Figure 1}

A normal B mild C moderate D severe E T2WI manifestation. A. A Newborn with a birth weight of $3.05 \mathrm{~kg}$, normal delivery at 36+3 weeks and clear amniotic fluid, Apgar score of 1 '-9', 5 '-9'. The serum total bilirubin was $502.3 \mathrm{~mol} / \mathrm{L}$ on the 6th day after birth. Routine MRI of the basal ganglia examined on the 9th day after birth showed an equal signal or slightly higher signal on T1WI, and the signal of globus pallidus was slightly higher than that of other nuclei in the basal ganglia on T1WI. 